\title{
A Cloud TOPSIS Method for Multiple Criteria Decision Making with Interval Number
}

\author{
Tie-Dan Wang \\ Institute of Quality Development, Kunming University of \\ Science and Technology, 650093, Kunming, PR China
}

\section{Xiao-Shi Shao}

Institute of Quality Development, Kunming University of Science and Technology, 650093, Kunming, PR China xiaoshi_likeyue@sohu.com

\author{
Ding-Hong Peng \\ Institute of Quality Development, Kunming University of \\ Science and Technology, 650093, Kunming, PR China \\ pengdinghong2006@163.com
}

\begin{abstract}
In order to ensure the accuracy of decision-making, need to be integrated the inevitable uncertainty information and fuzzy information into their decision-making process. In view of this, this paper proposed a Cloud TOPSIS method with the decision matrix contained the uncertainty and fuzziness information on the basis of the cloud drops and cloud drops distribution. In order to mine the data deeply, it converted the interval number model to the cloud model by establishing correspondence between the cloud drops and the endpoints. Next, the novel and key technique of the Cloud TOPSIS method including normalized the cloud decision matrix, compared the size of two cloud variables and calculated the distance measure. Finally, a numerical example is given, and the result was compared with the interval TOPSIS method result, demonstrating the feasibility and effectiveness of the proposed Cloud TOPSIS method.
\end{abstract}

Keywords-TOPSIS; Cloud model; Multicriteria decision making; Distance measures

\section{INTRODUCTION}

Within the multiple criteria decision making (MCDM), many realistic situations such as the complexity of real world, time pressure, lack of knowledge or data about the problem domain and information or the decision makers (DMs) have limited attention and information processing capacities, make it less and less possible for DMs to give exact estimations of single value, but interval values. Indeed, the interval numbers are the suitable, convenient and simple method of operation expression. DMs only provide two numbers to express the decision parameters, and it has been utilized for most investigation already. But there are some shortcomings, such as using interval numbers only mined the two endpoints of the data value, other most numerical information among the interval being ignored. What's more, the ignored information will affect the final decision deeply.

The Cloud model [2-7] defined by $\mathrm{Li}$ et al. is a cognitive model that mainly reflects the uncertainties of things in the universe and the concepts in human knowledge. The uncertainties mentioned above mainly include the fuzziness, randomness, and the association between them. Thus far, the normal Cloud model based on normal distribution and
Gaussian MF is the most applicable one. It has been successfully applied to many fields, such as data mining [5,8], uncertainty reasoning [4,9]; time series prediction $[10,11]$. However, the various theories mentioned above are directly utilized the cloud model as a data processing model for the problem.

TOPSIS method was initially proposed by Hwang and Yoon(1981) [13,14], The basic idea of the TOPSIS method is that the chosen alternative should have the shortest distance from the positive ideal solution (PIS) and the farthest distance from the negative ideal solution (NIS). As various types of uncertainties and imprecision often exist, the information of alternatives are vague, imprecise and uncertain by nature, a variety of more in-depth-study of a new approach emerged, such as those reported in Lai et al. (1994), Chen (2000), Abo-Sinna \& Amer (2005), Shih et al. (2007) and Shih (2008), Abo-Sinna et al. (2008) [12-16]. To improve the data-accuracy of the method become the new darling of researchers. As mentioned, in this paper we put forward an approach about extended TOPSIS, called the Cloud TOPSIS Method (C-TOPSIS). It is based on the concepts of the Cloud model and the general framework of TOPSIS method, modified the extent of data mining and more closer to the real mean what the DMs want to express.

\section{REVIEW OF ClOUd MODEL AND OpERATION}

In this section, we give some brief background information, such as the normal cloud model[7], the approach about comparing the size of two cloud-variables and the approach about calculating the distance measure of cloud. Those definitions will be employed in the C-TOPSIS Method below.

Definition 1 [2,3] (Clouds and Clouds droplet) Suppose $U$ is a quantitative domain with numerical expression; $C$ is the concept of characterization on $U$, if the value of $x \in U$ is a random realization of $C$, the certainty of $\mathrm{x}$ to $C$ that is $\mu(x) \in[0,1]$ will be a random number with stable tendency: $\mu: U \rightarrow[0,1], \forall x \in U, x \rightarrow \mu(x)$, and the distribution of $x$ on domain $U$ is known as clouds, written as clouds $C(X)$. Each of $\mathrm{x}$ is known as a clouds 
droplet [1]. If the domain corresponding to concept is the ndimensional space, it can be extended to n-dimensional clouds. In theory, there are several forms of cloud model, but the normal cloud model is commonly used in practice.

Definition 2 [6] (Normal Cloud Method) Assume that $U$ is a quantitative numerical universe of discourse and $C$ is the qualitative concept in $U$. If the quantitative value $x \in U$, and $x$ is a random achievement of the qualitative concept $C$. If $x$ meets: $x \sim N\left(E x, E n^{\prime 2}\right)$, where $E n^{\prime} \sim N\left(E n, H e^{2}\right)$, and the confirmation degree for which $x$ belongs to $C$ is:

$$
\mu=e^{-\frac{(x-E x)^{2}}{2\left(E n^{\prime}\right)^{2}}} .
$$

Then we call the distribution of $x$ on the domain $U$ is the one-dimension normal cloud.

The Cloud model can describe the overall quantitative property of a concept by three numerical characteristics, namely, Ex, En, and He. Ex is the mathematical expectation of the Cloud drops belonging to a concept in the universe. It is the most representative and typical sample of the qualitative concept. En represents the uncertainty measurement of a qualitative concept. It is determined by both the randomness and the fuzziness of the concept. On the one hand, as the measurement of randomness, En reflects the dispersing extent of the Cloud drops, which is similar to the standard deviation of a random variable. On the other hand, as the measurement of fuzziness, it represents the scope of the universe that can be accepted by the concept. He is the uncertainty degree of En, also seen as the entropy of entropy. He reflects the dispersion of the Cloud drops. The larger the He is, the larger its dispersion is, the larger the randomness of degree of membership is, and the larger the thickness of the Cloud is.

The one-dimension normal Cloud is the most basic tool to express the language value [12], and can be generated by the Cloud's digital character $(E x, E n, H e)$. The mathematic expectation curve (MEC) of one-dimension normal Cloud to a linguistic concept is:

$$
\operatorname{MEC}(x)=\exp \left[-(x-E x)^{2} / 2(E n)^{2}\right]
$$

Definition 3 [16] (The Operation Rules of Cloud) Given any two clouds $C_{1}$ and $C_{2}$, their digital characters are $C_{1}\left(E x_{1}, E n_{1}, H e_{1}\right)$ and $C_{2}\left(E x_{2}, E n_{2}, H e_{2}\right)$, respectively, then their arithmetic operations are defined as follows:

(1) The digital characters of $C_{1}+C_{2}$ are $\left(E x_{1}+E x_{2}, \sqrt{E n_{1}^{2}+E n_{2}^{2}}, \sqrt{H e_{1}^{2}+H e_{2}^{2}}\right)$;

(2) The digital characters of $C_{1}-C_{2}$ are $\left(E x_{1}-E x_{2}, \sqrt{E n_{1}^{2}+E n_{2}^{2}}, \sqrt{H e_{1}^{2}+H e_{2}^{2}}\right)$;

(3) The digital characters of $C_{1} \times C_{2}$ are :

$\left(E x_{1} \times E X_{2},\left|E x_{1} E x_{2}\right| \times \sqrt{\left(\frac{E n_{1}}{E x_{1}}\right)^{2}+\left(\frac{E n_{2}}{E x_{2}}\right)^{2}},\left|E x_{1} E x_{2}\right| \times \sqrt{\left(\frac{H e_{1}}{E x_{1}}\right)^{2}+\left(\frac{H e_{2}}{E x_{2}}\right)^{2}}\right)$
To rank the clouds, we give a method to compare the size of two Cloud-variables, and the method as follows.

Definition 4 (The Compare Rules of Cloud) Let $C_{i}$ and $C_{j}$ are two clouds, and their digital characters are $C_{i}=\left(E x_{i}, E n_{i}, H e_{i}\right)$ and $C_{j}=\left(E x_{j}, E n_{j}, H e_{j}\right)$, respectively, then

(1) If $E x_{i}>E x_{j}$, then $C_{i}$ is bigger than $C_{j}$, denoted by $C_{i}>C_{j}$

(2) If $E x_{i}=E x_{j}$, and $E n_{i}<E n_{j}$, then $C_{i}$ is bigger than $C_{j}$, denoted by $C_{i}>C_{j}$,

(3) If $E x_{i}=E x_{j}$, and $E n_{i}=E n_{j}$, then

(1) if $H e_{i}<H e_{j}$, then $C_{i}$ is bigger than $C_{j}$, denoted by $C_{i}>C_{j}$,

(2) if $H e_{i}=H e_{j}$, then $C_{i}$ and $C_{j}$ are identical, denoted by $C_{i}=C_{j}$.

We provide an approach to measure the distance between two cloud-variables. The distance measure is an important concept of Cloud TOPSIS Method which we will introduce below, and the concrete approach as follow.

Definition 5 (The Distance between Clouds) Let $C_{i}$ and $C_{j}$ be two clouds, and their digital characters are $C_{i}=\left(E x_{i}, E n_{i}, H e_{i}\right)$ and $C_{j}=\left(E x_{j}, E n_{j}, H e_{j}\right)$, respectively, then the distance measure between $C_{i}$ and $C_{j}$ is defined as:

$$
d\left(C_{i}, C_{j}\right)=1 / 4\left(\left|\left(E x_{i}-E x_{j}\right)-\left(E n_{i}-E n_{j}\right)\right|+\left|\left(E x_{i}-E x_{j}\right)+\left(E n_{i}-E n_{j}\right)\right|\right) \times\left(H e_{i}+H e_{j}\right)
$$

It is easy to proof the distance has the following properties:

1. $0 \leq d\left(C_{i}, C_{j}\right) \leq 1$;

2. $d\left(C_{i}, C_{j}\right)=d\left(C_{j}, C_{i}\right)$;

3. $d\left(C_{i}, C_{i}\right)=0$.

\section{Cloud TOPSis MethodOlOGY}

Suppose a MCDM problem has $m$ alternatives, and $n$ decision criteria/attributes, $C_{1} \ldots C_{n}$. Every alternative is evaluated with respect to the $n$ criteria /attributes. Every alternatives for each criterion is given a values/ratings expressed by interval values and form a decision matrix denoted by $A=\left[a_{i j}\right]_{m \times n}(i=1,2, \cdots m ; j=1,2, \cdots n)$, where $a_{i j}{ }^{L}$ and $a_{i j}{ }^{U}$ are the lower and upper bounds of it. Then $X_{i}=\left[a_{i 1}, a_{i 2}, \cdots, a_{i n}\right](i=1,2, \cdots m)$ is a discrete set of alternatives, and $C_{j}=\left[a_{1 j}, a_{2 j}, \cdots a_{m j}\right]^{T}(j=1,2, \cdots n)$. In the process of solving practical problems, the degree of emphasis on different criteria may be different, therefore need to set the corresponding weight $w_{j}(j=1,2, \cdots n)$, emphasized that $w_{j} \in[0,1], \sum_{j=1}^{n} w_{j}=1$. Based on the idea of TOPSIS method, this investigation present a Cloud TOPSIS Method 
(C-TOPSIS) which modify the interval-number expressed by the cloud model, then make the decision matrix to the Cloud Decision Matrix. In order to facilitate the comparing of data value in the next operation, need to convert into the same property type from the different, and eliminate the impact of values property of dimensions. It is data normalization processing and all concepts mentioned above are carried out as following:

Step 1. The value of each criterions for each alternative which DMs gave are represented by interval number. Convert the interval decision matrix $\left[a_{i j}\right]_{m \times n}=\left[a_{i j}{ }^{L}, a_{i j}{ }^{U}\right]_{m \times n}$ where have two element-variables, to a cloud decision matrix $\left[C_{i j}\right]_{m \times n}=\left[E x_{i j 0}, E n_{i j 0}, H e_{i j 0}\right]_{m \times n}$ where have three element-variables, according to the following expressions:

$$
\left\{\begin{array}{c}
E x_{i j 0}=\left(a_{i j}{ }^{L}+a_{i j}{ }^{U}\right) / 2 \\
E n_{i j 0}=\left(a_{i j}{ }^{U}-a_{i j}{ }^{L}\right) / 6 \\
H e_{i j 0}=\left(\left|\max _{1 \leq i \leq m} E n_{i j 0}-\min _{1 \leq i \leq m} E n_{i j 0}-2 E n_{i j 0}\right|\right) / 3
\end{array} .\right.
$$

where $E x_{i j 0}$ expresses the expectation of the interval values $a_{i j}$ and it is representative of its values by qualitative judgment; $E n_{i j 0}$ structured is based on the fact that interval $\left[E x_{i j 0}-3 E n_{i j 0}, E x_{i j 0}+3 E n_{i j 0}\right]$ best represents the qualitative judgment(99.74\%, 3 En rule), thus, 6 En can be used to reflect the extent of the fuzziness which exist the expression on expectation of the interval number; $H e_{i j}$ shows the extent of the fuzziness of the $E n_{i j}$ expressed the fuzziness of actual value.

Step 2. The different criterions value exist different dimensions and different value properties. In order to eliminate this adverse impact need to do data normalization processing. Through normalization, the element-variables of cloud model are between 0 to 1 , and retain varieties of properties and relation of the original data.

When the cloud variable of the decision matrix is the benefit attributes, normalized by:

$$
\left\{\begin{array}{c}
E x_{i j}=\frac{a_{i j}{ }^{L}+a_{i j}{ }^{U}-2 \min _{1 \leq i \leq m}\left\{a_{i j}{ }^{L}\right\}}{2\left(\max _{1 \leq i \leq m}\left\{a_{i j}{ }^{U}\right\}-\min _{1 \leq i \leq m}\left\{a_{i j}{ }^{L}\right\}\right)} \\
E n_{i j}=\frac{a_{i j}{ }^{U}-a_{i j}{ }^{L}}{6\left(\max _{1 \leq i \leq m}\left\{a_{i j}{ }^{U}\right\}-\min _{1 \leq i \leq m}\left\{a_{i j}{ }^{L}\right\}\right)} \\
H e_{i j}=\frac{\left|\max _{1 \leq i \leq m} E n_{i j}-\min _{1 \leq i \leq m} E n_{i j}-2 E n_{i j}\right|}{3}
\end{array} .\right.
$$

And when the cloud variable of the decision matrix is the cost attributes, normalized by:

$$
\left\{\begin{array}{c}
E x_{i j}=\frac{2 \max _{1 \leq i \leq m}\left\{a_{i j}{ }^{U}\right\}-a_{i j}{ }^{L}-a_{i j}{ }^{U}}{2\left(\max _{1 \leq i \leq m}\left\{a_{i j}{ }^{U}\right\}-\min _{1 \leq i \leq m}\left\{a_{i j}{ }^{L}\right\}\right)} \\
E n_{i j}=\frac{a_{i j}{ }^{U}-a_{i j}{ }^{L}}{6\left(\max _{1 \leq i \leq m}\left\{a_{i j}{ }^{U}\right\}-\min _{1 \leq i \leq m}\left\{a_{i j}{ }^{L}\right\}\right)} \\
H e_{i j}=\frac{\left|\max _{1 \leq i \leq m} E n_{i j}-\min _{1 \leq i \leq m} E n_{i j}-2 E n_{i j}\right|}{3}
\end{array}\right.
$$

Step 3. Determine the positive ideal solution and the negative ideal solution of the Cloud-model TPOSIS as the following expression:

$$
\begin{aligned}
C^{+}=\max C_{. j}^{+}(j=1,2, \ldots, n)=\left(E x_{. j}^{+}, E n_{. j}^{+}, H e_{. j}^{+}\right)(j=1,2, \ldots, n) \\
\quad C^{-}=\min _{. j}^{-}(j=1,2, \ldots, n)=\left(E x_{. j}^{-}, E n_{. j}^{-}, H e_{. j}^{-}\right)(j=1,2, \ldots, n) .
\end{aligned}
$$

where the positive ideal solution and the negative ideal solution are determined according to the maximum and minimum from all values/ratings of the each criteria /attributes, and the max and min on the basis of definition 4 above-mentioned in section 2 .

Step 4. Calculate the distance from each value of cloud decision matrix to the PIS and NIS which are from each criteria/attributes, using the following method:

$$
\begin{gathered}
d_{i j}^{+}=\left\{\begin{array}{l}
d\left(C_{i j}, C_{. j}^{+}\right)=1 / 4\left(\left|\left(E x_{i j}-E x_{. j}^{+}\right)-\left(E n_{i j}-E n_{. j}^{+}\right)\right|\right. \\
\left.+\left|\left(E x_{i j}-E x_{. j}^{+}\right)+\left(E n_{i j}-E n_{. j}^{+}\right)\right|\right) \times\left(H e_{i j}+H e_{. j}^{+}\right)
\end{array} .\right. \\
d_{i j}^{-}=\left\{\begin{array}{l}
d\left(C_{i j}, C_{. j}^{-}\right)=1 / 4\left(\left|\left(E x_{i j}-E x_{. j}^{-}\right)-\left(E n_{i j}-E n_{. j}^{-}\right)\right|\right. \\
\left.+\left|\left(E x_{i j}-E x_{. j}^{-}\right)+\left(E n_{i j}-E n_{. j}^{-}\right)\right|\right) \times\left(H e_{i j}+H e_{. j}^{-}\right)
\end{array}\right.
\end{gathered}
$$

Among $i=1,2, \ldots, m, j=1,2, \ldots, n$.

Step 5. Calculate the PIS and NIS of each alternative under every criteria/attributes, by the following way:

$$
\begin{aligned}
& S_{i}^{+}=S\left(C_{i .}, C^{+}\right)=\sum_{j=1}^{n} w_{j} d\left(C_{i j}, C_{. j}^{+}\right) \\
& S_{i}^{-}=S\left(C_{i}, C^{-}\right)=\sum_{j=1}^{n} w_{j} d\left(C_{i j}, C_{. j}^{-}\right) .
\end{aligned}
$$

$w_{j}$ represents the weight of the impact on decision about it criteria/attributes.

Step 6. Calculate the relative closeness to the ideal solution and rank the alternatives. The relative closeness of the alternative $S_{i}$ can be expressed as:

$$
S_{i}=S_{i}^{-} /\left(S_{i}^{-}+S_{i}^{+}\right),(i=1,2, \ldots, m)
$$

\section{NUMERICAL EXAMPLE AND DISCUSSIONS}

In this section, we apply an example which adapted from [16] to illustrate our foregoing proposed method's outstanding effectiveness.

\section{A. Select Management Example Apply the Proposed Method}

An enterprise need select an outstanding management personnel from five candidates, in terms of the uniform rules to convert four evaluation criteria of them to the interval number expressed decision maker's subjective judgment. Assuming that five candidates expressed by 
$\left(x_{1}, x_{2}, x_{3}, x_{4}, x_{5}\right)$ and the four evaluation criteria as: Criteria 1 is work attitude and work style; Criteria 2 is cultural level and knowledge structure; Criteria 3 is leadership; Criteria 4 is develop the ability and innovative spirit. Therefore, there is an interval-valued $5 \times 4$ decision matrix presented as follow:

$\left[\begin{array}{llll}{[3,7]} & {[7,9]} & {[5,5]} & {[3,5]} \\ {[3,5]} & {[1,7]} & {[5,9]} & {[3,9]} \\ {[7,9]} & {[3,5]} & {[5,7]} & {[1,5]} \\ {[3,5]} & {[5,7]} & {[3,7]} & {[7,9]} \\ {[7,9]} & {[3,7]} & {[5,9]} & {[3,7]}\end{array}\right]$

Step 1. Convert the interval values to the cloud decision matrix by eq.(4) in the third section mentioned. The converted results are form of the matrix as:

$$
\begin{gathered}
{\left[\begin{array}{ll}
{[5,0.000,0.222]} & {[4,0.333,0.000]} \\
{[7,0.667,0.222]} & {[6,1.000,0.444]} \\
{[6,0.333,0.000]} & {[3,0.667,0.222]} \\
{[5,0.667,0.222]} & {[8,0.333,0.000]} \\
{[7,0.667,0.222]} & {[5,0.667,0.222]}
\end{array}\right]} \\
{\left[\begin{array}{ll}
{[5,0.667,0.333]} & {[8,0.333,0.000]} \\
{[4,0.333,0.111]} & {[4,1.000,0.444]} \\
{[8,0.333,0.111]} & {[4,0.333,0.000]} \\
{[4,0.333,0.111]} & {[6,0.333,0.000]} \\
{[8,0.333,0.111]} & {[5,0.667,0.222]}
\end{array}\right]}
\end{gathered}
$$

Step 2. As these four criterion are the efficiency property variate, then normalized the cloud decision matrix by eq.(5) in the third section mentioned, and the converted normalized matrix as:

$$
\begin{gathered}
{\left[\begin{array}{ll}
{[0.333,0.111,0.056]} & {[0.875,0.042,0.000]} \\
{[0.167,0.056,0.019]} & {[0.375,0.125,0.056]} \\
{[0.833,0.056,0.019]} & {[0.375,0.042,0.000]} \\
{[0.167,0.056,0.019]} & {[0.625,0.042,0.000]} \\
{[0.833,0.056,0.019]} & {[0.500,0.083,0.028]}
\end{array}\right]} \\
{\left[\begin{array}{ll}
{[0.333,0.000,0.037]} & {[0.375,0.042,0.000]} \\
{[0.667,0.111,0.037]} & {[0.625,0.125,0.056]} \\
{[0.500,0.056,0.000]} & {[0.250,0.083,0.028]} \\
{[0.333,0.111,0.037]} & {[0.875,0.042,0.000]} \\
{[0.667,0.111,0.037]} & {[0.500,0.083,0.028]}
\end{array}\right]}
\end{gathered}
$$

Step 3. Determine the positive ideal solution and the negative ideal solution of the cloud-model TPOSIS according to the eq.(7) and eq.(8) above, and the results are shown:

$$
\begin{aligned}
C^{+}= & \left\{\max \left(C_{1}^{+}, C_{2}^{+}, C_{3}^{+}, C_{4}^{+}\right)\right\} \\
= & \{[0.833,0.056,0.019],[0.875,0.042,0.000], \\
& {[0.667,0.111,0.037],[0.875,0.042,0.000]\} } \\
C^{-}= & \left\{\min \left(C_{1}^{-}, C_{2}^{-}, C_{3}^{-}, C_{4}^{-}\right)\right\} \\
= & \{[0.167,0.056,0.019],[0.375,0.125,0.056], \\
& {[0.333,0.111,0.037],[0.250,0.083,0.028]\} }
\end{aligned}
$$

Step 4. Calculate the distance from each value of cloud decision matrix to the PIS which are from each criteria/attributes by eq.(9). The calculation results are as shown in Table 1:

TABLE 1. Solving Individual Distance to the Positive IDEAL SOLUTION

\begin{tabular}{|c|l|l|l|l|}
\hline Candidate & $d_{i 1}^{+}$ & $d_{i 2}^{+}$ & $d_{i 3}^{+}$ & $d_{i 4}^{+}$ \\
\hline$x_{1}$ & 0.01852 & 0.00000 & 0.01235 & 0.00000 \\
\hline$x_{2}$ & 0.01235 & 0.01389 & 0.00000 & 0.00694 \\
\hline$x_{3}$ & 0.00000 & 0.00000 & 0.00309 & 0.00868 \\
\hline$x_{4}$ & 0.01235 & 0.00000 & 0.01235 & 0.00000 \\
\hline$x_{5}$ & 0.00000 & 0.00521 & 0.00000 & 0.00521 \\
\hline
\end{tabular}

Calculate the PIS of every alternative under every criteria/attributes according to eq.(11) in the Step 5. We assume that Criteria 1 and Criteria 4's weights each of three tenths of overall judgment, and other two criteria distribute the remaining weights on average. Should be emphasized that the sum of all weights must be one, such as . Specific results are as follows:

$w_{1}=0.3 ; w_{2}=0.2 ; w_{3}=0.2 ; w_{4}=0.3$

$S_{1}^{+}=0.00802469 ; S_{2}^{+}=0.00856481 ; S_{3}^{+}=0.00322145 ; S_{4}^{+}=0.0061728 ; S_{5}^{+}=0.00260416$

Step 5. Calculate the distance from each value of cloud decision matrix to the NIS which are from each criteria/attributes by eq.(10). The calculation results are as shown in Table 2:

TABLE 2. Solving IndiVidual Distance To The Negative IDEAL SOLUTION

\begin{tabular}{|c|l|l|l|l|}
\hline Candidate & $d_{i 1}^{-}$ & $d_{i 2}^{-}$ & $d_{i 3}^{-}$ & $d_{i 4}^{-}$ \\
\hline$x_{1}$ & 0.006173 & 0.013889 & 0.004115 & 0.001736 \\
\hline$x_{2}$ & 0.000000 & 0.000000 & 0.012346 & 0.015625 \\
\hline$x_{3}$ & 0.012346 & 0.002315 & 0.003086 & 0.000000 \\
\hline$x_{4}$ & 0.000000 & 0.006944 & 0.000000 & 0.008681 \\
\hline$x_{5}$ & 0.012346 & 0.005208 & 0.012346 & 0.006944 \\
\hline
\end{tabular}

Calculate the NIS of every alternative under every criteria/attributes according to eq.(12) in the Step 5. We assume that Criteria 1 and Criteria 4's weights each of three tenths of overall judgment, and other two criteria distribute the remaining weights on average. Should be emphasized that the sum of all weights must be one, such as . Specific results are as follows:

$$
\begin{aligned}
& w_{1}=0.3 ; w_{2}=0.2 ; w_{3}=0.2 ; w_{4}=0.3 \\
& S_{1}^{-}=0.00597350 ; S_{2}^{-}=0.00715663 ; S_{3}^{-}=0.00478395 ; S_{4}^{-}=0.00399305 ; S_{5}^{-}=0.0092978
\end{aligned}
$$

Step 6. Calculate the relative closeness to the ideal solution as follow: 
$S_{1}=0.42673403 ; S_{2}=0.45521472 ; S_{3}=0.59759036 ; S_{4}=0.39278937 ; S_{5}=0.78119935$

In this case, the rank of the alternatives in descending order is $S_{5}>S_{3}>S_{2}>S_{1}>S_{4}$.

\section{B. Comparison Analysis}

Through the above example we get the result is same as using the interval TOPSIS method. But the extent of precision using the Cloud TOPSIS Method is more highly than using interval data directly. Then we list the relative closeness by two different methods, the Cloud TOPSIS method (C-TOPSIS) and the internal TOPSIS method (ITOPSIS) as shown in Table 3:

Table 3. The Relative Closeness by Two Different Methods (PRECISION: 0.001)

\begin{tabular}{|c|c|c|c|c|c|}
\hline Candidate & $S_{1}$ & $S_{2}$ & $S_{3}$ & $S_{4}$ & $S_{5}$ \\
\hline $\begin{array}{c}\text { C-TOPSIS } \\
\text { Method }\end{array}$ & 0.428 & 0.455 & 0.598 & 0.393 & 0.781 \\
\hline $\begin{array}{c}\text { I-TOPSIS } \\
\text { Method }\end{array}$ & 0.467 & 0.467 & 0.535 & 0.416 & 0.717 \\
\hline
\end{tabular}

Due to the fact that, obviously, using interval TOPSIS method occurred the same relative closeness from two candidates led to cannot choose the better from them. Because of interval number only mined the two endpoints of the data value, other most numerical information among the interval being ignored and the ignored information is the key to differ from both two candidates. The Cloud TOPSIS Method can elicit more valuable information of intervals and avoid occurring this problem. Therefore it is more effective and reasonable.

It demonstrate that the method put forward above is more fully mining the implicit data information to make the results more accurate closer to the real situation. Meanwhile the numerical example is also provided to illustrate the Cloud TOPSIS Method is suitable for solving MCDM problems with uncertainty information. The results have showed that using C-TOPSIS Method to measure the weight value of every alternative is an encouraging and robust method for solving the MCDM problems with uncertainty information.

\section{CONCLUSIONS}

In this paper, focus in the MCDM problems express by interval number, we propose a new Cloud TOPSIS methods to solve. Because the cloud model get the utmost out of the information of data rather than the interval number only mined the two endpoints value, In this case, it will better to adapt the uncertainty environment including fuzziness and randomness, and more accurately reflect the true idea of DMs. For TOPSIS algorithm, the biggest advantage is to find the optimal solution by stepwise approach the positive ideal solution and far away from the negative ideal solution, can obtain an acceptable compromise solution to decisionmakers, called satisfactory solution. Therefore, we provide the Cloud TOPSIS method to make the information dug deeper and expressed more accurate as well as to get the satisfactory solution closer the real situation. As we structure the C-TOPSIS method, we give the approach to modify the interval numbers to the cloud model variables, to normalize the cloud model variables, to compare the size of two cloud variables and to calculate the distance measure. Through the numerical example, it illustrates that the C-TOPSIS Method has the higher accuracy and data mining of information than interval model expressed. The results have showed that using C-TOPSIS Method to solve the MCDM problems with uncertainty information is an encouraging and robust method.

\section{ACKNOWLEDGMENTS}

We greatly acknowledge the financial support provided by the Natural Science Foundation of China (Grant No.61364016), China Postdoctoral Science Foundation (Grant No, 2015T80990 and No, 2014M550473), and the Applied Basic Research Programs of Yunnan Province, China (No. 2014FB136).

\section{REFERENCES}

[1] Li, D. F.: An Approach to Fuzzy Multi-Attribute Decision Making under Uncertainty. Information Sciences. 169, 97-112 (2005)

[2] Wang, D., Zeng, D., Singh, V. P., Xu, P. C.: A Multidimension Cloud Model-Based Approach for Water Quality Assessment. Environmental Research. 149, 113--121 (2016)

[3] Whaiduzzaman, M., Gani, A., Anuar, N. B., Shiraz, M., Haque, M. N., Haque, I. T.: Cloud Service Selection Using Multicriteria Decision Analysis. The Scientific World Journal. 2014, 1--10 (2014)

[4] Parhoudeh, S., Baziar, A., Mazareie, A., Kavousi-Fard, A.: A Novel Stochastic Framework Based on Fuzzy Cloud Theory for Modeling Uncertainty in The Micro-Grids. Electrical Power and Energy Systems. 80, 73--80 (2016)

[5] Liu, D. D., Chen, X. H., Lou, Z. H.: Analysis on Characteristics of Spatial-temporal Precipitation Distribution Based on Cloud Model (in Chinese with English abstract). J. Hydraul. Eng. 40, 850--857 (2009)

[6] Li, D. Y., Du, Y.: Artificial Intelligence with Uncertainty. J. Software. 15, (2004)

[7] Li, D., Liu, C., Gan, W.: A New Cognitive Model: Cloud Model. International Journal of Intelligent Systems. 24, 357-375 (2009)

[8] Wang, H., Deng, Y.: Spatial clustering method based on cloud model. IEEE 4th International Conference on Fuzzy Systems and Knowledge Discovery. 2, 272--276 (2007)

[9] Chen, H., Li, B.: Qualitative Rules Mining and Reasoning Based on Cloud Model. IEEE 2nd International Conference on Software Engineering and Data Mining. 523--526 (2010)

[10] Jiang, R.; Li, D., Chen, H.: Time-series Prediction with Cloud Models in DMKD. Lecture Notes in Computer Science. 1574, 525--530 (1999)

[11] Zhang, J., Hu, G.: Application of Uncertainty Reasoning Based on Cloud Model in Time Series Prediction. Journal of Zhejiang University (Science A). 4, 578--583 (2003)

[12] Abo-Sinna, M. A., Amer, A. H.: Extensions of TOPSIS for Multi-objective Large-scale Nonlinear Programming Problems. Applied Mathematics and Computation. 162, 243$-256(2005)$ 
[13] Abo-Sinna, M. A., Amer, A. H., Ibrahim, A. S.: Extensions of TOPSIS for Large Scale Multi-Objective Non-Linear Programming Problems with Block Angular Structure. Applied Mathematical Modelling. 32, 292--302 (2008)

[14] Chen, C. T.: Extensions of the TOPSIS for Group DecisionMaking Under Environment. Fuzzy Sets and Systems, 114, 1--9 (2000)

[15] Shih, H.S., Shyur, H. J., Lee, E. S.: An Extension of TOPSIS for Group Decision Making. Mathematical and Computer Modelling. 45, 801--813 (2007)

[16] Shih, H. S.: Incremental Analysis for MCDM with an Application to Group TOPSIS. European Journal of Operational Research. 186, 720--734 (2008) 